\title{
The CVD paradox: mortality vs prevalence
}

A 1l-cause mortality is in decline-indeed, worldwide, life expectancy increased by 9 years between 1979 and 2004 (Beaglehole, R. \& Bonita, R. Lancet 372, 1988-1996; 2008). Although cardiovascular disease (CVD) is still the leading cause of death, age-related CVD mortality has also been falling over the past 25-30 years. Numerous studies suggest that risk-factor reductions and treatment of established disease each account for approximately $40-60 \%$ of the decline in CVD mortality, with another $0-10 \%$ attributable to undetermined causes. Unless the incidence of CVD decreases in step with the decline in mortality over the next 20-30 years, we will experience a rise in the prevalence of CVD.

This latter phenomenon has also occurred in the recent past. For the period 1995-2005, the incidence of first diagnosis of coronary heart disease decreased in the UK, but the prevalence of coronary heart disease increased; investigators concluded that this discrepancy resulted from a substantially greater decrease in agerelated mortality among patients with coronary heart disease, compared with the decrease in incidence of this condition (Davies, A. R. et al. Eur. Heart J. 28, 21422147; 2007). Additionally, CVD prevalence worldwide is expected to increase further as a result of the epidemic of obesity and its consequences, including diabetes, hypertension and dyslipidemia. So, one of life's amazing paradoxes is that the prevalence of CVD is increasing despite great advances in technology and treatment and reductions in age-related CVD deaths. Consequentially, we will have to endure significant economic burden. The solution to the paradox is clear: promote cardiovascular health and prevent CVD.

Health-care costs are increasing worldwide, mostly because of the use of important but expensive new technologies and treatments, but also as a result of people surviving for longer and, therefore, requiring more health care (including hospitalization) over their lifetime. To avoid the substandard quality of life and the costly technologies and treatments associated with CVD, we must focus our attention on low-cost strategies that promote cardiovascular health and prevent CVD. In the UK, part of the $62 \%$ decrease in rate of incidence of first myocardial infarction between 1979 and 2004 can

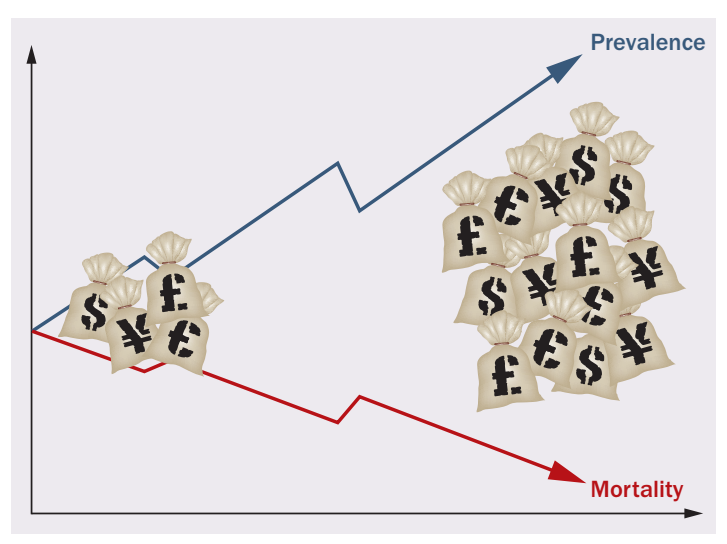

be accounted for by changes in smoking habits, systolic blood pressure and levels of HDL and non-HDL cholesterol (Hardoon, S. L. et al. Circulation 117, 598-604; 2008). Obesity, diabetes, hypertension, hyperlipidemia and tobacco consumption are all major risk factors for CVD that are cheap and easy to prevent. Indeed, WHO estimates of the cost of implementing and running programs related to tobacco control and reduction of salt consumption range from US $\$ 0.40$ to US $\$ 1.00$ per person per year, and recommend increased taxes on tobacco, alcohol and sugary drinks, which would not only deter people from consuming large quantities of these items-a step in the right direction for promoting health and preventing CVD-but also provide money with which to implement prevention programs (Beaglehole, R. \& Bonita, R. Lancet 372, 1988-1996; 2008).

We must make major inroads in the promotion of cardiovascular health and prevention of CVD over the next few years. If we do not increase our efforts, the worldwide increase in life expectancy and the consequential aging of the population will result in more people developing CVD and requiring treatment for their condition at some point over the course of their lifetime. The associated high health-care costs may make provision of modern technology and treatments to such a vast number of people unfeasible.

doi:10.1038/nrcardio.2009.187

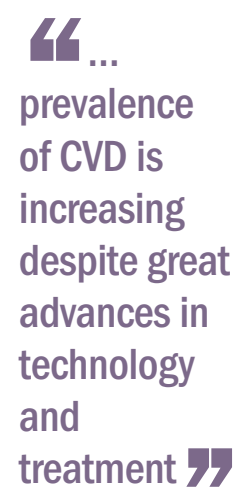

Valentin Fuster is the Editor-in-Chief and Bryony M. Mearns is the Editor of Nature Reviews Cardiology.

Competing interests The authors declare no competing interests. 